\title{
ESTÁGIO DE ENTRADA DE UMA UPS ON-LINE DE ALTA EFICIÊNCIA CONTROLADO POR UM ÚNICO DSP
}

\author{
Luciano Schuch \\ Universidade Luterana do Brasil - ULBRA \\ CEP: 97020-001 - Santa Maria, RS - Brasil \\ lschuch@ieee.org - http://www.ulbra.br
}

\author{
Cassiano Rech, José R. Pinheiro \\ Universidade Federal de Santa Maria - UFSM \\ CEP 97105-900, Santa Maria, RS, BRASIL, \\ renes@ctlab.ufsm.br - http://www.ufsm.br/gepoc
}

\begin{abstract}
This paper proposes an integrated ZVT auxiliary commutation circuit applied to the pre-regulator converter and to the battery charger of Uninterruptible Power Systems (UPS). With the proposed integrated ZVT auxiliary commutation circuit there are few auxiliary components and the overall efficiency increases. In addition, two control strategies, one analog control scheme and one digital control scheme, are analyzed, and performance and cost of these strategies are compared. The analog control system is implemented with several dedicated IC's whereas the digital control system is implemented with a low cost single-chip DSP controller. A $580 \mathrm{~W}$ closed-loop prototype, with universal input, has been implemented to evaluate the performance of the proposed system.
\end{abstract}

KEYWORDS: Soft-switching, digital control, battery charger, universal input, UPS.

\section{RESUMO}

Neste artigo, é proposta a utilização de um único circuito de auxílio à comutação ZVT, aplicado tanto no estágio préregulador como no carregador de baterias de Sistemas Ininterruptos de Energia (UPS). Também, é demonstrado que a utilização de uma única célula de comutação ZVT permite que se obtenha, além da redução do número de componentes auxiliares, um melhoria no rendimento do conversor. Adicionalmente, duas estratégias de controle, digital e analógica, são analisadas e comparadas do ponto de vista de

\footnotetext{
Artigo Submetido em 25/02/03

1a. Revisão em 21/10/05;

Aceito sob recomendação do Editor Consultor

Prof. Dr. José Antenor Pomilio
}

desempenho e custo. O sistema de controle analógico é implementado com vários Cl's dedicados, já o controlador digital é implementado por meio de um único DSP de baixo custo. Um protótipo em malha fechada de $580 \mathrm{~W}$, com entrada universal, operando em $100 \mathrm{kHz}$, foi implementado nã só para validar o circuito de auxílio à comutação, mas também para avaliar o desempenho do sistema proposto.

PALAVRAS-CHAVE: comutação suave, controle digital, carregador de baterias, entrada universal, UPS.

\section{INTRODUÇÃO}

A utilização maciça de sistemas informatizados, em aplicações consideradas críticas, tais como Bancos, hospitais, sistemas de telecomunicação e de segurança, entre outros, tem aumentado a necessidade de fontes ininterruptas de energia (UPS - Uninterruptible Power System), que fornecem energia com boa qualidade e apresentam uma elevada estabilidade e confiabilidade. Entre as principais características desejáveis de uma UPS, pode-se citar: elevado fator de potência, grande autonomia do banco de baterias, elevada densidade de potência, além de reduzida taxa de distorção harmônica na tensão de saída.

Para atender às normas internacionais referentes ao conteúdo harmônico de corrente injetado na rede pública de energia (IEC-61000-3-2, 2000), um conversor préregulador pode ser incluído no estágio de entrada da UPS. Por apresentar características bastante favoráveis para este tipo de aplicação, o conversor boost tem sido preferido (Todd, 1996).

Outro fator de considerável importância é a escolha e projeto do banco de baterias, a ser utilizado em uma UPS, uma vez que grande parte dos custos, volume e peso desses sistemas correspondem ao já citado estágio. Assim, uma 
atenção especial deve ser dada na especificação do banco de baterias, visando à maximização de sua vida útil e à redução de seu custo.

No que se refere à densidade de potência do estágio de entrada de uma UPS, a adequada definição da estrutura a ser utilizada no estágio do carregador de baterias, bem como a utilização de técnicas de comutação suave, tanto para o conversor pré-regulador como para o carregador de baterias, assume uma importância fundamental (Schuch et al, 2002).

No intuito de atender os requisitos acima mencionados para o estágio de entrada de uma UPS, neste trabalho é proposta a utilização de um único circuito de auxílio à comutação ZVT (Zero Voltage Transition), aplicado tanto no estágio pré-regulador quanto no carregador de baterias da UPS. O conversor pré-regulador é do tipo boost, que opera no modo de condução contínua (CCM), com entrada universal. Para o carregador de baterias, é proposta a utilização de um conversor bi-direcional (buck + boost), que permite uma maior flexibilidade na escolha da tensão do banco de baterias, possibilitando não só uma significativa redução de custo, peso e volume, mas ainda uma melhoria no método de carga das baterias. A utilização de uma técnica de comutação suave permite a operação em freqüências de chaveamento mais elevadas, possibilitando, com isso, uma redução significativa no peso e no volume dos elementos magnéticos presentes no sistema, além de uma melhor resposta dinâmica.

Para o adequado funcionamento do conversor e para a regulação das variáveis de interesse do sistema proposto, foram estudas duas implementações para as malhas de controle, uma analógica, outra digital. Essas implementações foram analisadas e comparadas do ponto de vista de performance e custo.

\section{DESCRIÇÃO DO CONVERSOR PROPOSTO}

A Figura 1 mostra o conversor bidirecional e o conversor pré-regulador (conversor boost operando em modo de condução contínua - CCM), com entrada universal (85 $\mathrm{V}_{\text {ef }}$ a $265 \mathrm{~V}_{\text {ef }}$ ), que compõem o estágio de entrada da UPS sob análise. Um circuito de auxílio à comutação ZVT foi incluído nesse sistema, com o objetivo de elevar a

Tabela 1. Modos de operação do conversor bidirecional.

\begin{tabular}{cccc}
\hline ModO & DESCRIÇÃO & $\begin{array}{c}\text { ESTADO } \\
\text { DA REDE }\end{array}$ & $\begin{array}{c}\text { TENSÃO DO BANCO } \\
\text { DE BATERIAS }\end{array}$ \\
\hline Modo I & Boost & Falha & $>1,75 \mathrm{~V} / \mathrm{e}$ \\
Modo II & $\begin{array}{c}\text { Buck: modo } \\
\text { corrente } \\
\text { Muck: modo } \\
\text { tensão }\end{array}$ & $O k$ & $<2,30 \mathrm{~V} / \mathrm{e}$ \\
& & & $>2,45 \mathrm{~V} / \mathrm{e}$ \\
\hline
\end{tabular}

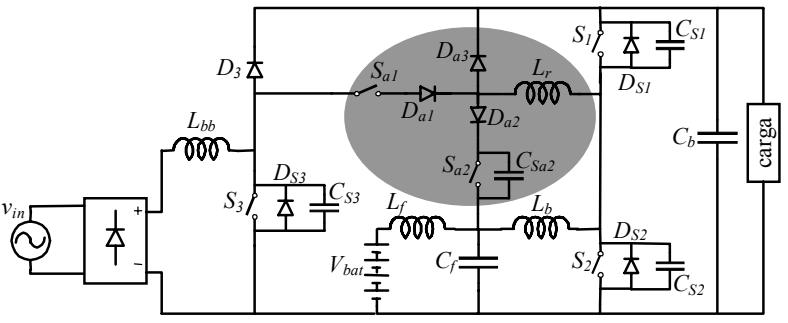

Figura 1. Circuito proposto.

eficiência e reduzir a interferência eletromagnética.

O conversor pré-regulador é composto por um retificador em ponte completa, um indutor $\left(L_{b b}\right)$, um diodo $\left(D_{3}\right)$, um interruptor $\left(S_{3}\right)$ e um capacitor $\left(C_{b}\right)$.

O conversor bidirecional é composto por dois interruptores $\left(S_{1}\right.$ e $\left.S_{2}\right)$, dois diodos $\left(D_{S 1}\right.$ e $\left.D_{S 2}\right)$ e um filtro T, composto por dois indutores $\left(L_{b}\right.$ e $\left.L_{f}\right)$ e um capacitor $C_{f}$. Esse conversor bidirecional pode operar como:

- Conversor boost $\left(S_{2}-D_{S I}\right)$, responsável por suprir energia para a carga, caso haja uma falha da rede pública de energia (modo I).

- Conversor buck $\left(S_{l}-D_{S 2}\right)$, responsável pela carga do banco de baterias (modo II e modo III).

O conversor buck pode operar em dois modos distintos, que dependem do nível de tensão do banco de baterias. Se a tensão do banco de baterias for menor que a tensão de flutuação (tensão para manter o estado de carga completa), o conversor buck opera no modo corrente (modo II) para recarregar as baterias. Se a tensão do banco de baterias atingir a tensão de $2,45 \mathrm{~V}$ por elemento (para baterias de tipo VRLA - Valve Regulated Lead Acid), o conversor buck troca do modo corrente para o modo tensão (modo III), assim mantem o estado de carga completa. Os modos de operação do conversor bidirecional podem ser vistos na Tabela 1.

Cabe dizer que o CAC é composto por dois interruptores $\left(S_{a 1}\right.$ e $\left.S_{a 2}\right)$, três diodos auxiliares $\left(D_{a 1}, D_{a 2}\right.$, e $\left.D_{a 3}\right)$ e um indutor ressonante $L_{r}$. O princípio de operação do CAC é discutido na próxima seção deste artigo.

\section{CIRCUITO DE AUXÍLIO À COMUTAÇÃO}

Com a inclusão do CAC, mostrado na Figura 1, todos os interruptores principais apresentam comutação suave do tipo ZVT, já os interruptores auxiliares a do tipo ZVS (ZeroVoltage Switching) e ZCS (Zero-Current Switching). O CAC tem volume reduzido, pois apenas um indutor ressonante é usado, para obter comutação suave nos três interruptores principais em todos os modos de operação; além disso os níveis de corrente média e eficaz que 
circulam pelo CAC são reduzidos. Os modos de operação do conversor proposto são descritos a seguir.

\subsection{Modo I (boost)}

Como mostrado na Tabela 1, o modo boost ocorre quando a rede pública de energia estiver fora dos padrões aceitáveis e o conversor bidirecional estiver fornecendo energia para carga. As principais formas de ondas teóricas do processo de comutação suave, neste modo, são apresentadas na Figura 2. Para que o interruptor principal $S_{2}$ entre em condução em ZVT, primeiramente, deve-se acionar o interruptor auxiliar $S_{a 2}$, que inicia a etapa de carga linear da corrente do indutor $L_{r}$. No instante que $i_{L r}$ for igual a $I_{\text {bat(dis), inicia-se a }}$ ressonância entre a energia do capacitor $C_{S 2}$ e a energia do indutor $L_{r}$, levando a tensão sobre o interruptor $S_{2}$ a zero. Posteriormente, bloqueia-se o interruptor auxiliar $S_{a 2}$ (ZVS), para iniciar a descarga linear da corrente do indutor $L_{r}$.

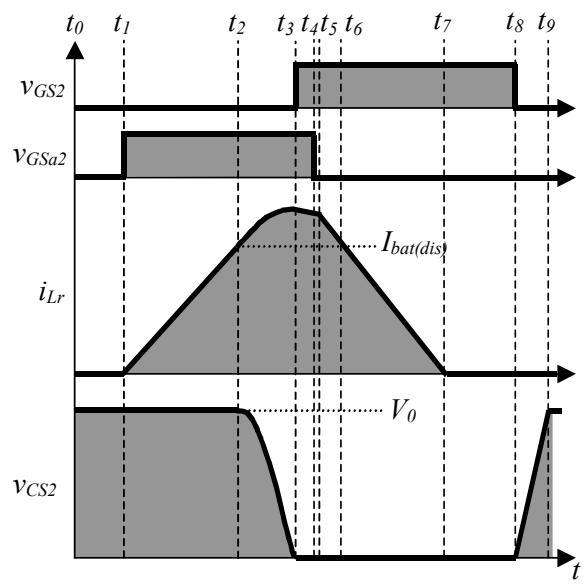

Figura 2. Principais formas de ondas teóricas do modo I.

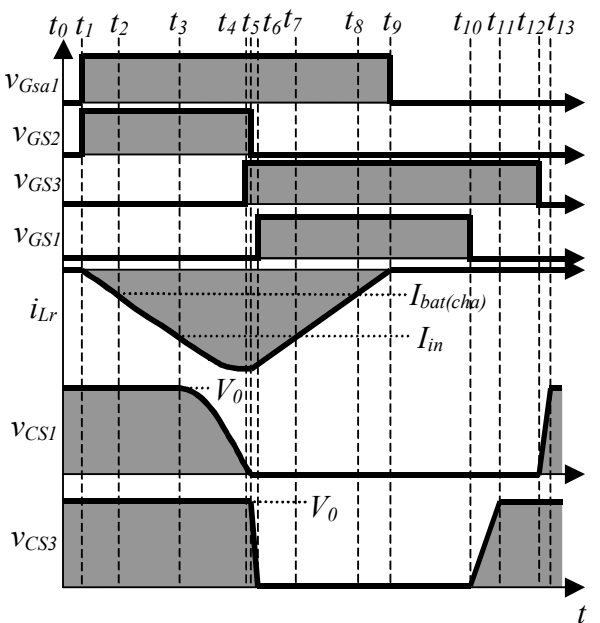

Figura 3. Principais formas de ondas teóricas dos modos II e III.

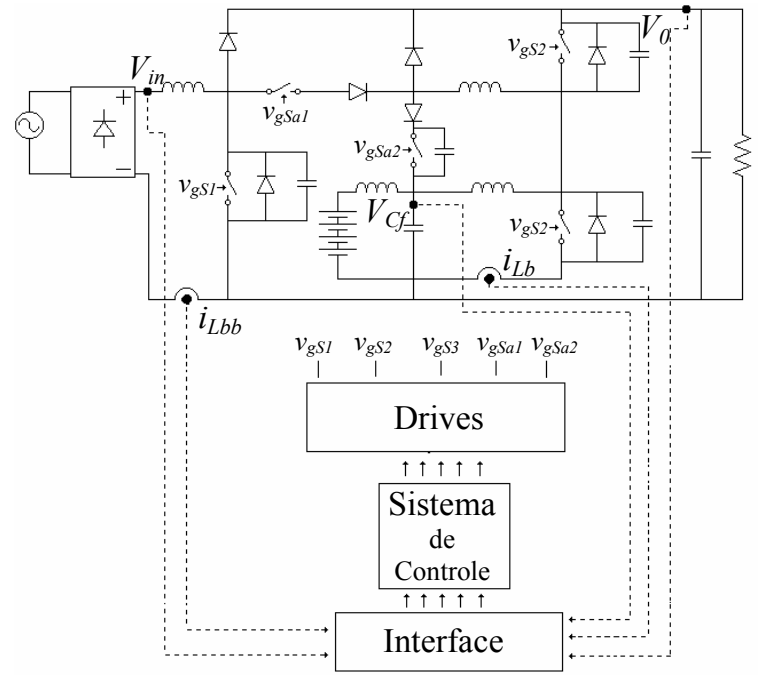

Figura 4. Circuito esquemático do sistema de controle.

\subsection{Modo II e Modo III (pré-regulador + buck)}

O modo II e o modo III ocorrem quando a rede pública de energia estiver dentro dos padrões aceitáveis. Em função disso, o conversor pré-regulador mantém o barramento $\mathrm{CC}$ e o conversor buck carrega o banco de baterias, no modo corrente ou no modo flutuação. Para garantir a entrada em condução dos interruptores principais $S_{1}$ e $S_{3}$ em ZVT, devese acionar os interruptores $S_{a l}$ e $S_{2}$, para iniciar a carga linear da corrente do indutor $L_{r}$. No instante em que $i_{L r}$ for igual a $I_{i n}$, inicia-se a ressonância entre o capacitor $C_{S 3}$ e o indutor $L_{r}$, que leva a tensão sobre o interruptor $S_{3}$ a zero; assim, o diodo $D_{S 3}$ entra em condução. A partir deste instante, o interruptor $S_{2}$ pode ser bloqueado para que ocorra uma segunda ressonância entre o indutor $L_{r}$ e os capacitores $C_{S 1}$ e $C_{S 2}$, que leva a tensão sobre o interruptor $S_{1}$ a zero. As principais formas de ondas teóricas são apresentadas na Figura 3.

A metodologia de projeto do $\mathrm{CAC}$ e do conversor bidirecional é realizada de acordo com Schuch et al (2002, 2004(a) e 2004(b)) e o conversor pré-regulador (580W) é projetado conforme Todd (1996).

\section{SISTEMA DE CONTROLE}

O controle das variáveis de interesse é implementado por meio de duas diferentes tecnologias: uma, analógica com CI's dedicados (UC3854 para o conversor pré-regulador e UC3524 para os demais conversores); outra, digital com um único DSP de baixo custo. As quatro malhas de controle, que devem controlar a carga do banco de baterias, regular a tensão de saída (barramento CC) e garantir elevado fator de potência, são vistas na Figura 4. 


\subsection{Controle Analógico}

Como mencionado anteriormente, a carga do banco de baterias apresenta dois modos distintos: o de corrente e o de tensão (ou flutuação). O diagrama de blocos destes dois modos são mostrados na Figura 5(a) e na Figura 5(b), respectivamente.

Para o modo tensão, optou-se por um controlador proporcional, porque é simples e atende a especificação imposta a este modo (tensão de flutuação recomendada de 2,15 a 2,23 Volts por elemento (V/e) com variação máxima de $1 \%$ ). Por outro lado, para o modo corrente, utilizou-se o controle da corrente média, porque ele garante que a corrente de carga do banco de baterias apresente erro nulo em, regime permanente, com boa resposta dinâmica, além de evitar oscilações subarmônicas (instabilidade) (Sun e Bass, 1999).

Para a obtenção do modelo matemático do conversor buck, no modo tensão e corrente, o banco de baterias foi substituído por um circuito equivalente, mostrado na Figura 6, baseado no modelo apresentado em Salamch et al (1992). O valor da resistência $R_{s}$ é obtido diretamente do catálogo dos fabricantes, e o valor de $R$ e $C$ são obtidos pelo do tempo de carga e pela corrente de fuga (autodescarga) das baterias.

$\mathrm{Na}$ Figura 5(a), $C_{1}(s)$ é a função de transferência do compensador proporcional, $G_{1}(s)$ é o modelo da planta e $H_{1}(s)$ é o ganho do sensor. A função de transferência $G_{1}(s)$ é dada por:

$$
G_{1}(s)=\frac{A_{0} s^{2}+A_{1} s+A_{2}}{B_{0} s^{4}+B_{1} s^{3}+B_{2} s^{3}+B_{3} s+B_{4}},
$$

sendo:

$$
\begin{aligned}
& A_{0}=V_{0} L_{f} C R ; \\
& B_{1}=C_{f} L_{f} L_{b}+C_{f} L_{b} R_{s} R C ; \\
& A_{1}=\left(L_{f}+R_{s} R C\right) V_{0} ; \\
& B_{2}=C_{f} L_{b} R+C_{f} L_{b} R_{s}+L_{b} R C+ \\
& +L_{f} C R \\
& A_{2}=\left(R+R_{s}\right) V_{0} ; \\
& B_{3}=R_{s} R C+L_{b}+L_{f} ; \\
& B_{0}=L_{f} C_{f} L_{b} R C ; \\
& B_{4}=R+R_{s}
\end{aligned}
$$

O projeto do compensador proporcional é realizado por meio da equação (2), baseado no teorema do valor final:

$$
K_{p} \geq V_{s} \frac{V_{\text {fref }}-\Delta V_{f} H_{1}}{\Delta V_{f} H_{1}^{2} V_{i \min }},
$$

sendo $V_{s}$ o ganho do modulador, $\Delta V_{f}$ a variação máxima da tensão de flutuação e $V_{\text {imim }}$ a mínima tensão do barramento CC.

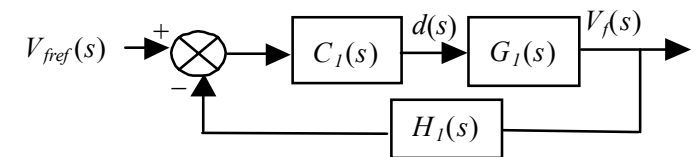

(a)

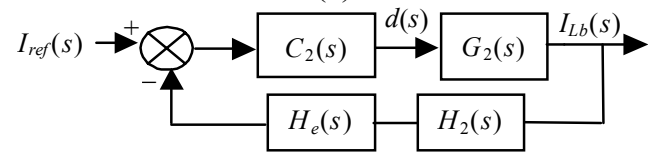

(b)

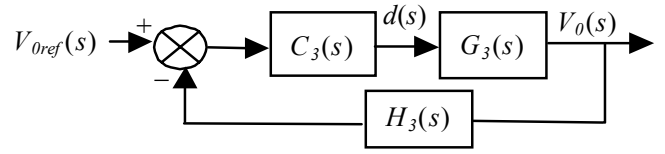

(c)

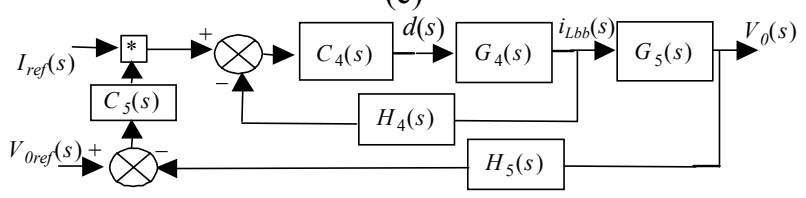

(d)

Figura 5. Diagrama de blocos: (a) Buck modo tensão; (b) Buck modo corrente; (c) Boost; (d) pré-regulador.

Dessa forma, considerando-se que o erro no valor da tensão de flutuação deve ser inferior a $1 \%\left(\Delta V_{f}\right)$, obtém-se $K_{p} \geq 20$.

$\mathrm{Na}$ Figura 5(b), $H_{e}(s)$ representa a efeito do chaveamento (Ridley, 1991); $G_{2}(s)$, o modelo da planta; $H_{2}(s)$, o ganho do sensor de corrente $\left(i_{L b}\right)$ e $C_{2}(s)$, o compensador de corrente, cujas funções de transferências são dadas por:

$$
\begin{gathered}
H_{e}(s)=\frac{s^{2}}{\left(\pi f_{s 1}\right)^{2}}-\frac{s}{2 f_{s 1}}+1, \\
G_{2}(s)=\frac{V_{i}(R C s+1)}{L_{b} R C s^{2}+\left(R_{s} R C+L_{b}\right) s+\left(R+R_{s}\right)}, \\
C_{2}(s)=\frac{K_{c}\left(1+s / \omega_{z}\right)}{s\left(1+s / \omega_{p}\right)} .
\end{gathered}
$$

O compensador de corrente $C_{2}(s)$ é projetado da seguinte maneira: o pólo de alta freqüência $\omega_{p}$ é posicionado na freqüência de chaveamento para filtrar a ondulação de corrente; em seguida, o zero $\omega_{z}$ é posicionado na metade da freqüência de corte do filtro de saída para estender a freqüência de cruzamento por zero; finalmente, o ganho do

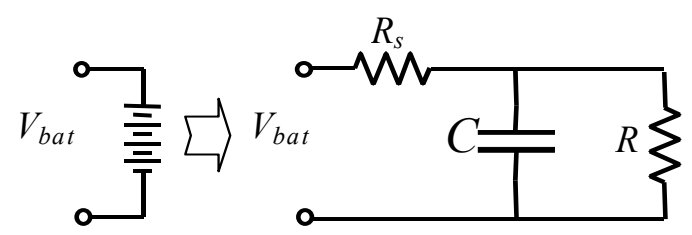

Figura 6. Circuito elétrico equivalente do banco de baterias. 


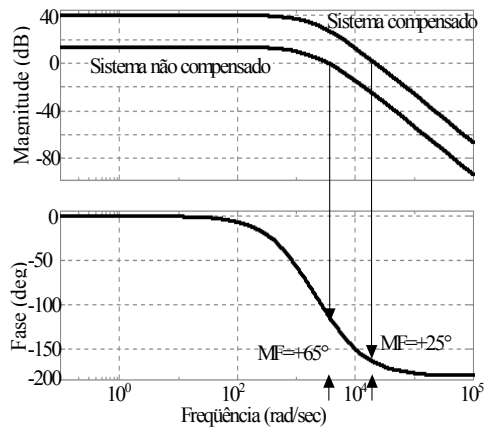

(a)

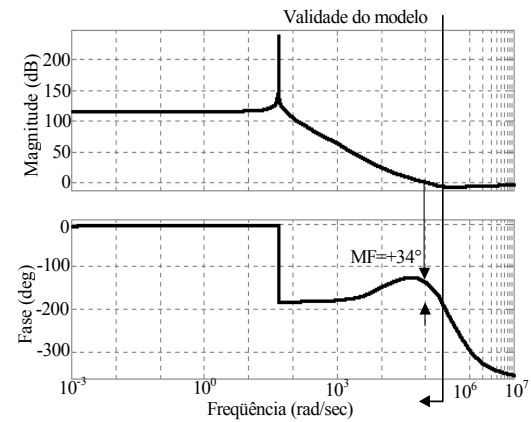

(b)

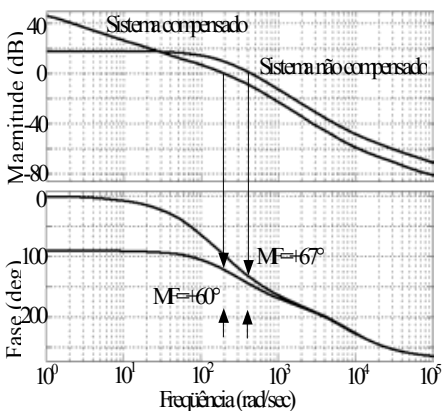

(c)

Figura 7. Diagrama de Bode: (a) Buck modo tensão; (b) Buck modo corrente; (c) Boost.

compensador, $K_{c}$ é calculado para evitar que o sinal de saída do compensador intercepte novamente o sinal de rampa, no período em que o interruptor está desligado (oscilação subharmônica), o que levaria o sistema à instabilidade. Assim, os parâmetros $\omega_{p}, \omega_{z}$ e $K_{c}$ do compensador de corrente são: $\omega_{p}=628320 \mathrm{rad} / \mathrm{s}, \omega_{z}=12566 \mathrm{rad} / \mathrm{s}$ e $K_{c}=4300$.

Os diagramas de Bode do conversor buck, no modo tensão e no modo corrente, estão expostos na Figura 7(a) e na Figura 7(b), respectivamente. A margem de fase do sistema compensado, no modo tensão, é de $+25^{\circ}$; já no modo corrente é de $+34^{\circ}$, comprovando-se a estabilidade dos sistemas. É importante salientar que a margem de fase de $+25^{\circ}$ é suficiente para garantir a estabilidade do sistema, porque a dinâmica da carga (banco de baterias) é lenta. Além disso, o efeito do pólo, na origem do compensador da corrente média, não é visualizado na Figura 7(b), pois a planta possui um zero $(1 / R C)$ muito próximo da origem. Outra característica deste diagrama de Bode é que ele só é valido até a metade da freqüência de comutação, devido à inclusão do efeito da comutação $\left(H_{e}(s)\right)$.

O controle da tensão de saída é realizado pela malha de tensão do conversor boost, Figura 5(c), ou pela malha de tensão do conversor pré-regulador, Figura 5(d). Como o objetivo das duas malhas de tensão é garantir que a tensão de saída $V_{0}$ seja regulada com erro nulo, optou-se por um controlador do tipo proporcional-integral (PI) para ambos os conversores.

O controle da tensão de saída através do conversor boost é definido a partir das seguintes funções de transferências: $G_{3}(s)$ é o modelo da planta, $C_{3}(s)$ é o compensador proporcional-integral e $H_{3}(s)$ é o ganho do sensor, cujas funções de transferências são dadas por:

$$
G_{3}(s)=\frac{-\frac{L_{e q} A}{R_{0}} s+\left(1-\frac{R_{e q}}{R_{0}}\right) A}{L_{e q} C_{b} \mathrm{~s}^{2}+\left(\frac{L_{e q}}{R_{0}}+R_{e q} C_{b}\right) \cdot s+\left(1-\frac{R_{e q}}{R_{0}}\right)}
$$

$$
C_{3}(s)=K_{P I} \frac{\left(s+z_{P I}\right)}{s}
$$

sendo:

$$
\begin{array}{cc}
A=\frac{V_{b a t} R_{0}}{\left(R_{e q}+R_{0}\right) \bar{d}^{2}} ; & L_{e q}=\frac{L_{b}}{\bar{d}^{2}} ; \\
R_{e q}=R_{s} / \bar{d}^{2} & \bar{d}=V_{b a t} / V_{0} .
\end{array}
$$

Como pode ser visto em (6), a função de transferência $G_{3}(s)$ apresenta um zero no semi-plano direito do plano $s$, ou seja, $G_{3}(s)$ é de fase não mínima. Porém, devido à presença da resistência série $R_{S}$ (Figura 8 ) do banco de baterias e da resistência série equivalente do indutor boost $\left(L_{b}\right)$, o efeito do zero, no semi-plano direito, é reduzido, o que possibilita aumentar a ganho do PI de tal modo que o sistema em malha fechada mantenha-se estável, com resposta dinâmica semelhante a do conversor pré-regulador.

A resposta dinâmica do conversor boost pode ser similar à resposta dinâmica do conversor pré-regulador, já que as especificações impostas aos dois conversores são as mesmas. Assim, diminui-se a banda passante do sistema em malha fechada para obter-se a margem de fase desejada, com uma resposta dinâmica similar a do conversor préregulador. Cabe dizer que o projeto do $C_{3}(s)$ é realizado da seguinte maneira: reduz-se a freqüência de cruzamento por zero do sistema em malha fechada para metade do sistema original e defini-se a margem de fase desejada $\left(60^{\circ}\right)$. Então, obtém-se: $K_{P I}=0,304$ e $z_{P I}=0,0117 \mathrm{rad} / \mathrm{s}$. O diagrama de Bode, desse modo, é visto na Figura 7(c), que mostra que o sistema compensado é estável, com margem de fase de $+60^{\circ}$.

Na Figura 8, é mostrada a posição dos pólos em malha fechada, variando-se o resistência $R_{S} \quad(0-1 \Omega)$ e considerando-se que os parâmetros do PI foram projetados para o valor nominal de $R_{s}(200 \mathrm{~m} \Omega)$. Nessa figura, pode-se verificar que o sistema em malha fechada se mantém estável, para valores de $R_{s}$ maiores que $22 \mathrm{~m} \Omega$. Para esta 
aplicação, foram utilizadas baterias UNIKOR 12V7AH, sendo a variação de $R_{s}$ aproximadamente $80 \%$ a $150 \%\left(0^{\circ} \mathrm{C}\right.$ a $40^{\circ} \mathrm{C}$ ) do seu valor nominal. Conseqüentemente, o sistema em malha fechada é estável para todo faixa de variação de $R_{s}$.

Finalizando, na Figura 5(d), é visto o sistema de controle do conversor pré-regulador (conversor boost em CCM), sendo $G_{4}(s)$ a função de transferência $I_{L b}(s) / d(s) ; G_{5}(s)$ a função de transferência $V_{0}(s) / I_{L b}(s) ; C_{4}(s)$ o compensador de corrente e $C_{5}(s)$ o compensador de tensão. As funções de transferências $G_{4}(s)$ e $G_{5}(s)$ são dadas por:

$$
\begin{gathered}
G_{4}(s)=R_{0}\left(\frac{V_{\text {in }}}{V_{0}}\right)^{2} /\left(C_{b} R_{0} s+1\right), \\
G_{5}(s)=\frac{V_{0} R_{0}}{L_{b b}+R_{0}} \cdot \frac{\left(s+\frac{2}{R_{0} C_{b}}\right)}{s^{2}+\frac{1}{R_{0} C_{b}} s+\frac{\left(V_{i n} / V_{0}\right)^{2}}{L_{b b} C_{b}}},
\end{gathered}
$$

Este sistema de controle possui uma malha de corrente interna rápida, que garante que a corrente de entrada seja senoidal e em fase com a tensão alternada de entrada. $C_{4}(s)$ é um compensador da corrente média, como o apresentado na equação (5), sendo seu projeto realizado de forma similar. A malha externa de tensão regula a tensão de saída por intermétio da referência especificada. $C_{5}(s)$ é um compensador proporcional-integral que, em malha fechada, apresenta uma pequena banda passante, em vista disso, a corrente de entrada apresenta uma baixa distorção.

\subsection{Controle Digital}

Existem, em geral, dois modos de projetar um controlador digital. O primeiro consiste em discretizar o modelo contínuo da planta e, então, projetar o controlador diretamente no domínio discreto. O segundo, um dos modos mais utilizados industrialmente para projetar um controlador digital, consiste em projetar o controlador no domínio contínuo e, após, faz uso de um método de discretização para obter semelhante desempenho (Rabbath e Nori, 2002).

\subsubsection{Método de Discretização}

Diversos métodos de discretização podem ser utilizados para converter um controlador analógico em um digital, tal como: Método de Discretização de Euler (Backward Euler), Transformação Bilinear, Resposta Invariante ao Impulso, entre outros.

O Método de Discretização de Euler é fácil de implementar e apresenta uma resposta equivalente a do controlador analógico. Assim, ele é um método recomendado para discretizar controladores analógicos aplicados a conversores estáticos de potência (Duan e Jin, 1999). Nesse método, a relação entre a variável $s$ do domínio contínuo e a variável $z$ do domínio discreto é dada por:

$$
s=(z-1) /\left(T_{s} z\right),
$$

onde $T_{s}$ é o período de amostragem.

\subsubsection{Leis de controle digital}

Aplicando-se o Método de Discretização de Euler nos controladores analógicos, apresentados anteriormente, é possível obter as leis de controle digital equivalentes, apresentadas na Tabela 2. É importante salientar que cada malha de controle possui sua própria referência (ref $[k])$ e sua variável de saída $(y[k])$; já o valor de $e[k]$ e $M[k]$ são obtidos por:

$$
\begin{gathered}
e[k]=\operatorname{ref}[k]-y[k], \\
M[k]=M[k-1]+e[k] .
\end{gathered}
$$

Pode-se observar, no entanto, que as variáveis de controle $(u[k])$ dependem do valor atual do erro $(e[k])$. Logo, se o tempo gasto para implementar o algoritmo de controle não for pequeno, pode-se obter um melhor desempenho ao predizer-se o sinal de saída $(y[k+1])$ no instante $t=k T_{s}$. A partir daí, é possível calcular o próximo valor da variável de

Tabela 2. Leis de controle digitais.

\begin{tabular}{l|c}
\hline Conversor & Controlador digital \\
\hline \multirow{3}{*}{ Buck } & Malha de corrente \\
& $u[k]=K_{11} e[k]+K_{21} T_{s} M[k]+K_{31} u[k-1]$ \\
& Malha de tensão \\
& $u[k]=K_{12} e[k]$ \\
\hline \multirow{2}{*}{$\begin{array}{l}\text { Pré- } \\
\text { regulador }\end{array}$} & $u[k]=K_{13} e[k]+K_{23} T_{s} M[k]+K_{33} u[k-1]$ \\
& Malha de tensão \\
& $u[k]=K_{14} e[k]+K_{24} T_{s} M[k]$ \\
\hline \multirow{2}{*}{ Boost } & Malha de tesnão \\
& $u[k]=K_{15} e[k]+K_{25} T_{s} M[k]$ \\
\hline
\end{tabular}

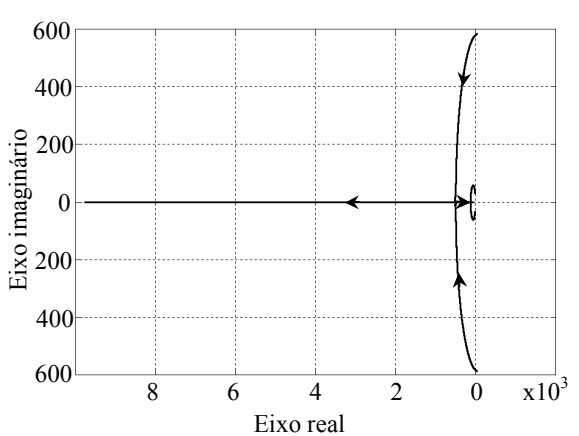

Figura 8. Posição dos pólos em malha fechada variando a resistência $R_{s}$. 
controle $(u[k+1])$. Em função disso, o seguinte algoritmo de predição linear (Bibian e Jin, 2000) foi usado para minimizar os atrasos computacionais:

$$
y[k+1]=2 y[k]-y[k-1]+A_{j} T_{s}(u[k]-u[k-1]),
$$

em que $A_{j}$ é uma constante que depende da planta a ser controlada. Uma predição do valor do sinal de saída, no fim do período de amostragem $(y[k+1])$, pode ser calculada com base nos valores atuais e nos passados da própria saída, esta somada a um termo de ajuste que consiste em valores presentes e passados da variável de controle $u$.

Para ilustrar a aplicação do algoritmo de predição, a lei de controle do conversor buck no modo corrente pode ser facilmente modificada, obtendo-se, então, a seguinte lei de controle preditiva:

$$
\begin{aligned}
& u[k+1]=K_{m} \operatorname{ref}[k+1]-K_{m}(2 y[k]-y[k-1]) \\
& +\left(K_{13}-A_{j} T_{s} K_{m}\right) u[k]+A_{j} T_{s} K_{m} u[k-1]+K_{21} T_{s} M[k]
\end{aligned}
$$

em que:

$$
K_{m}=K_{11}+K_{21} T_{s}
$$

A partir da equação (14), é possível calcular o valor da lei de controle para o próximo período de amostragem $(u[k+1])$, com base nos valores atuais e passados da saída e do valor da referência no próximo período de amostragem ref $[k+1]$ (gerado internamente pelo DSP). De modo similar, é possível obter as demais leis de controles preditivas.

\subsubsection{Freqüência de amostragem}

$\mathrm{O}$ aumento da freqüência de chaveamento possibilita reduzir os parâmetros dos filtros e melhorar a resposta dinâmica do sistema. Contudo, devido ao tempo de cálculo das leis de controle, não é possível aumentar a freqüência de amostragem (freqüência de atualização das leis de controle) na mesma taxa. Logo, a freqüência de chaveamento $\left(f_{s w}\right)$ pode ser aumentada se for mantida a mesma freqüência de amostragem $\left(f_{s}\right)$.

Como as leis de controle e o sistema de monitoramento (gerenciamento) são implementados em um único DSP (TMS320F241 da Texas Inst.), a freqüência de amostragem é limitada em $10 \mathrm{kHz}$. Por outro lado, as freqüências de chaveamento do conversor pré-regulador e do conversor buck foram definidas como $100 \mathrm{kHz}$, e a freqüência do conversor boost como $40 \mathrm{kHz}$ (Schuch et al, 2002). Conseqüentemente, existem 10 e 4 períodos de chaveamento em um período de amostragem, mostrados na Figura 9.

\subsubsection{Instante de amostragem}

A escolha do instante de amostragem é um fator importante para evita-se ruídos de medição na aquisição, causados pela

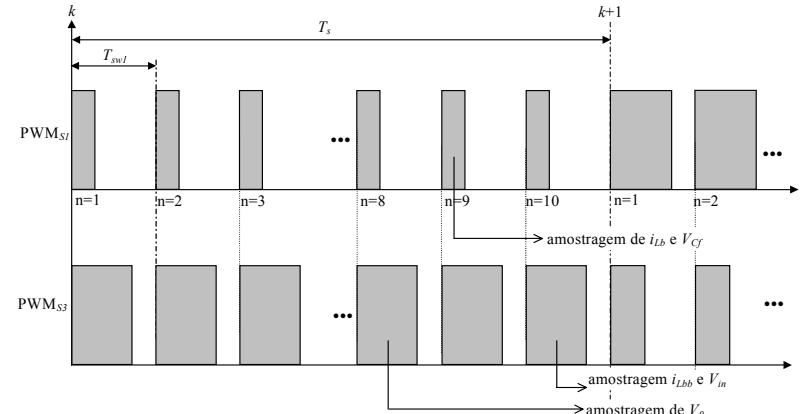

(a)

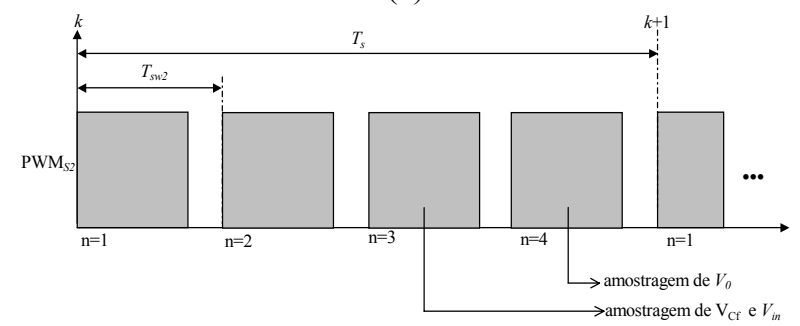

(b)

Figura 9. Sinal de comando dos interruptores principais e instantes de amostragem: (a) Modo II e III; (b) ModoI.

comutação dos interruptores, e para obter o valor médio aproximado das variáveis de interesse. A fim de obter o valor médio aproximado da corrente $i_{L b}$, é necessário amostrar essa corrente em um dos seguintes pontos (Prodic et. al., 2002):

$$
d_{S 1}[k] T_{s w 1} / 2 \text { ou }\left(1+d_{S 1}[k] T_{s w 1}\right) / 2
$$

O mesmo princípio pode ser usado para a corrente de entrada do conversor pré-regulador $\left(i_{L b b}\right)$. Por outro lado, as tensões $V_{0}, V_{i n}$ e $V_{C f}$ apresentam ondulações devido à comutação desprezíveis. Desse modo, não é necessário adquirir essas tensões em um ponto específico. Porém, devido aos ruídos de medição provenientes das comutações, é desejável amostrar esses sinais em instantes que não ocorram comutações dos interruptores ativos.

Devido a essas considerações, foi desenvolvido um algoritmo para o cálculo dos instantes de amostragem $\left(t_{s}\right)$ das tensões e das correntes. Assim, amostra-se o valor médio aproximado das variáveis de interesse, livres dos ruídos das comutações dos interruptores.

Por exemplo, o instante de amostragem da corrente $i_{L b}$ e da tensão $V_{C f}$ é calculado pela equação (17) e apresentado na Figura 9(a).

$$
t_{s}= \begin{cases}\frac{d_{S 1}[k] T_{s w 1}}{2} & \text { s se } d_{S 3}[k]>0.5 \\ \frac{\left(1+d_{S 1}[k]\right) T_{s w 1}}{2} & \text { se } d_{S 3}[k]<0.5\end{cases}
$$


O instante de amostragem das outras variáveis é obtido de forma similar. Finalmente, para os modos II e III, o valor da aquisição da tensão $V_{0}$ só é atualizado na lei de controle; quando a tensão de entrada for aproximadamente igual a zero $(120 \mathrm{~Hz})$, para se obter o valor médio da tensão de saída.

\section{RESULTADOS EXPERIMENTAIS}

Um protótipo em malha fechada foi construído em laboratório para comprovar o funcionamento do CAC e verificar o desempenho dos compensadores. Os parâmetros do protótipo são mostrados na Tabela 3 .

Na Figura 10, são apresentados os resultados experimentais que comprovam a comutação suave de todos os interruptores, para os três modos de operação. Na Figura 10(a) e na Figura 10(b), são apresentadas as comutações suaves dos interruptores $S_{2}$ e $S_{a 2}$, no modo I, respectivamente. Na Figura 10(c), (d), (e) e (f), são apresentadas as comutações suaves dos interruptores $S_{1}, S_{3}$, $S_{a 1}$ e $S_{2}$, no modo II, respectivamente. As comutações suaves dos interruptores presentes no modo III, são similares as do modo II.

Os rendimentos do conversor, no modo I e III, são vistos na Figura 11. Para o modo I, o rendimento para carga nominal foi de $94,6 \%$, enquanto para o conversor que opera sem o CAC, foi de $86 \%$. Conseqüentemente, a autonomia do banco de baterias é aumentada com a utilização do CAC. Para o modo II, o rendimento para carga nominal e tensão de entrada mínima foi de $96,4 \%$; para o conversor que opera sem o CAC, foi de $89,7 \%$. Já, o rendimento para a carga nominal e tensão de entrada de $110 \mathrm{~V}_{\text {ef }}$, foi de $96,5 \%$; para a tensão de entrada de $220 \mathrm{~V}_{\mathrm{ef}}$, foi de $97,2 \%$.

Os controladores analógicos foram implementados com CI's dedicados (um UC3854 para o conversor pré-regulador

Tabela 3. Parâmetros do Protótipo Implementado.

$\begin{array}{ll}\text { Tensão CA de entrada } & V_{\text {in }}=85 \text { to } 265 \mathrm{~V}_{\text {rms }}, f=60 \mathrm{~Hz} \\ \text { Tensão de saída } & V_{0}=400 \mathrm{~V} \\ \text { Tensão do banco de baterias } & V_{b a t}=48 \mathrm{~V} \\ \text { Potência de saída } & P_{0}=500 \mathrm{~W}(320 \Omega) \\ \text { Freqüência do buck } & f_{S 1}=100 \mathrm{KHz} \\ \text { Freqüência do boost } & f_{S 2}=40 \mathrm{KHz} \\ \text { Freqüência do pré-regulador } & f_{S 3}=100 \mathrm{KHz} \\ & C_{f}=1 \mathrm{mF} \\ \text { Filtro T } & L_{f}=1,6 \mu \mathrm{H} \\ & L_{b}=253 \mu \mathrm{H} \\ \text { Capacitor boost } & C_{b}=220 \mu \mathrm{F} \\ \text { Indutor boost (pré-regulador) } & L_{b b}=750 \mu \mathrm{H} \\ \text { Indutor ressonante } & L_{r}=53 \mu \mathrm{H} \\ \text { Capacitor } C_{S 3} & C_{S 3}=3.3 \mathrm{nF} \\ \text { Interruptores } S_{1}, S_{2} \text { e } S_{a 2} & \text { MOSFET IRFP450 } \\ \text { Interruptor } S_{a 1} & \text { IGBT G3N60C3D } \\ \text { Diodos } D_{a 1}, D_{a 2} \text { e } D_{a 3} & \text { MUR1560 } \\ \text { Diodo } D_{3} & \text { HFA25PB60 }\end{array}$

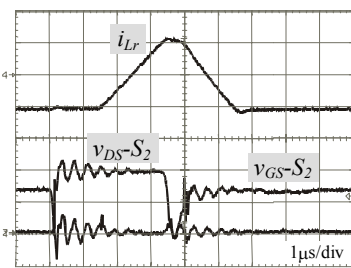

(a) $10 \mathrm{~A} / \mathrm{div}, v_{D}$

$200 \mathrm{~V} / \mathrm{div}$ e $v_{G S}$ $100 \mathrm{~V} / \mathrm{div}$

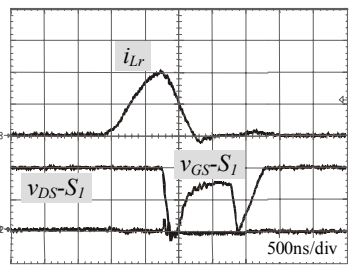

(c) $2 \mathrm{~A} / \mathrm{div}, v_{D S}$

$200 \mathrm{~V} / \mathrm{div}$ e $v_{G S}$ $100 \mathrm{~V} / \mathrm{div}$

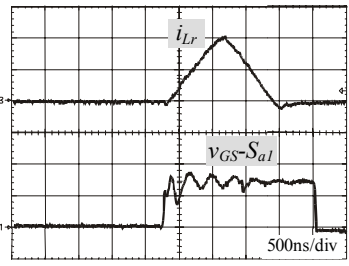

(e) $2 \mathrm{~A} / \mathrm{div}$ e $v_{G S}$ $100 \mathrm{~V} / \mathrm{div}$

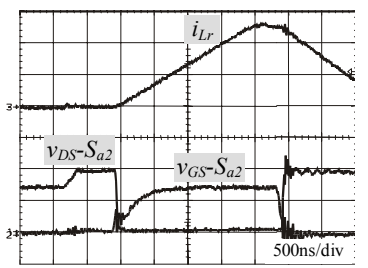

(b) $10 \mathrm{~A} / \mathrm{div}, v_{D S}$ $200 \mathrm{~V} / \mathrm{div}$ e $v_{G S}$ $100 \mathrm{~V} / \mathrm{div}$

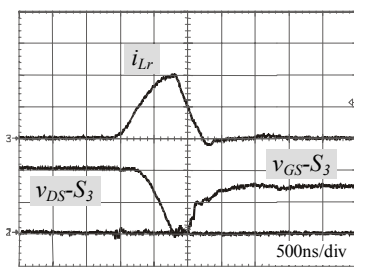

(d) $2 \mathrm{~A} / \mathrm{div}, v_{D S}$ $200 \mathrm{~V} / \mathrm{div}$ e $v_{G S}$ $100 \mathrm{~V} / \mathrm{div}$

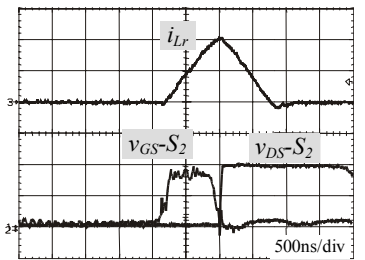

(f) $2 / \mathrm{div}, v_{D S} 200 \mathrm{~V} / \mathrm{div}$ $\mathrm{e}$ $v_{G S} 100 \mathrm{~V} / \mathrm{div}$
Figura 10. Resultados experimentais: comutação suave.

e dois UC3524 para os outros conversores). Por outro lado, os controladores digitais foram implementados com um único DSP de baixo custo (TMS320F421). Além disso, o sistema de monitoramento foi realizado no mesmo DSP por software; já no sistema analógico, foram utilizados diversos comparadores e portas lógicas para realizar a mesma função. $\mathrm{O}$ desempenho dos controladores em ambas as tecnologias (analógica e digital) é equivalente. Destaca-se a tecnologia digital pela flexibilidade de implementação via software, pela alta confiabilidade e pela facilidade de geração dos comandos dos interruptores auxiliares e, ainda, pela implementação do sistema de monitoramento em um único DSP.

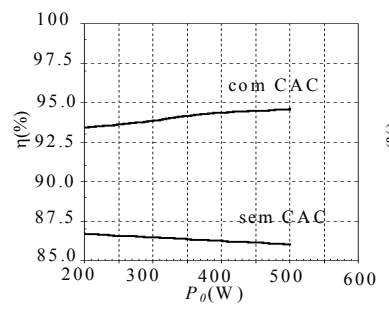

(a)

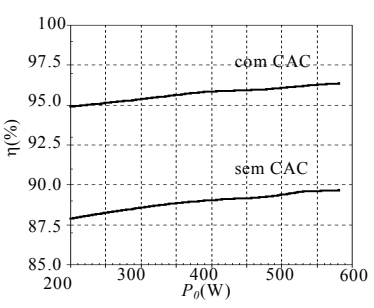

(b)
Figura 11. Rendimento do conversor proposto. 


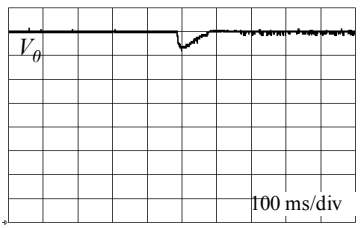

(a) controle analógico (50V/div e $2 \mathrm{~A} / \mathrm{div})$

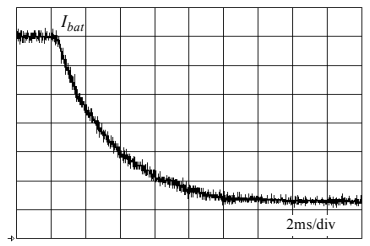

(c) controle analógico. $(0,2 \mathrm{~A} / \mathrm{div})$.

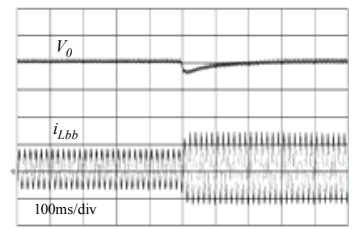

(e) controle analógico (100V/div e $5 \mathrm{~A} / \mathrm{div})$ (b) controle digital. $(50 \mathrm{~V} / \mathrm{div})$

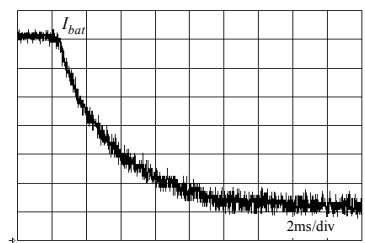
$(0,2 \mathrm{~A} / \mathrm{div})$.

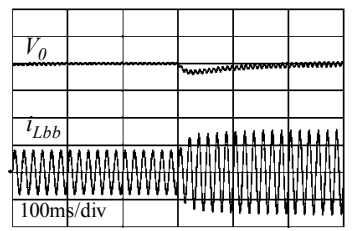

(e) controle digital (100V/div e 5A/div)

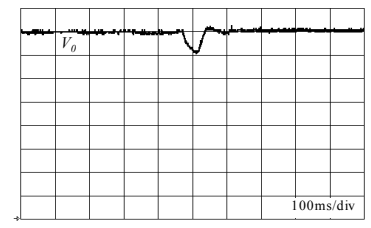

(d) controle digital

Figura 12. Desempenho dos controladores com perturbações externas.

A Figura 12(a) e a Figura 12(b) apresentam a resposta transitória do controlador analógico e do controlador digital, respectivamente, quando submetidos a um degrau de carga no modo I. Pode-se observar que as respostas são similares e atendem às especificações impostas ao conversor. A Figura 12(c) e a Figura 12(d) mostram a resposta transitória do conversor buck, quando ocorre uma transição do modo corrente para o modo tensão, que utiliza os controladores analógico e digital, respectivamente. Pode-se verificar que as respostas ocorrem sem oscilações e são muito semelhantes para as duas estratégias de controle.

Finalmente, para verificar o desempenho do conversor préregulador, nas duas técnicas de controle, foi realizado um degrau de carga no conversor, como mostrado na Figura 12(e) e na Figura 12(f). O fator de potência de entrada é, aproximadamente, igual a 0,98 e a THD da corrente de entrada é, aproximadamente, $6 \%$ para carga nominal e para toda faixa de variação da tensão de entrada, independentemente do tipo de controlador usado. A resposta transitória também é semelhante para os dois casos.

\section{CONCLUSÕES}

Um circuito de auxílio à comutação ZVT, aplicado ao estágio de entrada de uma UPS, foi apresentado e analisado; nele, os interruptores principais apresentam comutação do tipo ZVT e os interruptores auxiliares comutação do tipo ZCS e ZVS. O CAC utiliza poucos elementos, baixa energia reativa, fácil projeto e implementação. Vale resaltar que um indutor ressonante realiza a comutação suave nos três conversores que compõem o sistema.

A análise e o projeto do sistema de controle foram realizados e, ainda, a comparação da performance, utilizando-se duas diferentes tecnologias, analógica e digital. Ambas as técnicas de controle apresentaram respostas similares, atendendo às especificações impostas aos conversores. Contudo, a tecnologia digital é uma opção mais atrativa se comparada com a analógica, pois apresenta grande flexibilidade de implementação e alteração de projeto via software; alta confiabilidade; baixo custo de implementação em uma linha de produção; facilidade na geração dos sinais de comando dos interruptores auxiliares. Sem esquecer que o sistema de monitoramento (gerenciamento) de sistema pode ser implementado em um único DSP.

Para verificar o funcionamento do CAC proposto e o desempenho das estratégias de controle propostas, foi montado um protótipo de $580 \mathrm{~W}$.

\section{AGRADECIMENTOS}

Os autores agradecem ao CNPq e a CAPES pelo apoio financeiro e as empresas Icotron - an EPCOS Company, Toshiba do Brasil, Thorton Inpec LTDA e Texas Instruments pela doação de material de apoio.

\section{REFERÊNCIAS BIBLIOGRÁFICAS}

Bibian, S., Jin, H. (2000). Time Delay Compensation of Digital Control for DC Switchmode Power Supplies Using Prediction Techniques. IEEE Trans. Power Electr., 15(5): 835-842.

Duan, Y., Jin, H. (1999). Digital Controller Design for Switchmode Power Converters. Proc. IEEE APEC'99: 967-973.

International Electrotechnical Connission. (2000). IEC 61000-3-2: Electromagnetic Compatibility (EMC) Part 3: Limits - Section 2: Limits for Harmonic Current Emissions, $2^{\circ}$ edição.

Prodic, A., Chen, J., Erickson, R. W., Maksimovic, D. (2002). Digitally Controlled Low-Harmonic Rectifier Having Fast Dynamic Responses. Proc. IEEE APEC'2002: 476-482.

Rabbath, C. A., Hori, N. (2000). On a comparative study of digital redesign methods. IEEE Amer. Control Conf: 1154-1158. 
Ridley, R. B. (1991) A New, Continuous-Time Model For Current-Mode Control. IEEE Trans. Power Electr., 6(2): 271-278.

Salamch, Z. M., et al, (1992). A Mathematical Model for Lead-Acid Batteries in IEEE Trans. Energy Conv., 7(1): 93-97.

Schuch, L., Rech, C., Pinheiro, H., Hey, H. L., Gründling, H. A., Pinheiro, J. R. (2002). Analysis and Design of a New High-Efficiency Bi-Directional ZVT PWM Converter for DC Bus and Battery Bank Interface. Proc. IEEE APEC'2002: CD-ROM

Schuch, L., Rech, C., Pinheiro, H., Hey, H. L., Gründling, H. A., Pinheiro, J. R. (2004a). Análise e Projeto de um Conversor Bidirecional PWM de Alto Desempenho para Interface entre o Barramento $\mathrm{CC}$ e o Banco de Baterias. Revista Brasileira de Eletrônica de Potência (SOBRAEP), v.9, n.1, p.1-11, 2004

Schuch, L., Rech, C., Pinheiro, J. R. (2004b). Integrated ZVT Auxiliary Commutation Circuit for Input Stage of Double-Conversion UPSs. IEEE Transactions on Power Electronics, pp. 1486-1497, V 9, Nov, 2004.

Sum, J., Bass, R. M., (1999) Modeling and Practical Design Issues for Average Current Control. Proc. IEEE APEC'99: 980-986.

Todd, P. C., (1996) UC3854 Controlled Power Factor Circuit Design, Unitrode Application Note: 10-30310-322. 